\title{
Some facts from descriptive set theory concerning essential spectra and applications
}

\author{
by \\ Khalid Latrach (Aubière), J. Martin PaOli (Corte) \\ and Pierre Simonnet (Corte)
}

\begin{abstract}
Let $X$ be a separable Banach space and denote by $\mathcal{L}(X)($ resp. $\mathcal{K}(\mathbb{C}))$ the set of all bounded linear operators on $X$ (resp. the set of all compact subsets of $\mathbb{C}$ ). We show that the maps from $\mathcal{L}(X)$ into $\mathcal{K}(\mathbb{C})$ which assign to each element of $\mathcal{L}(X)$ its spectrum, approximate point spectrum, essential spectrum, Weyl essential spectrum, Browder essential spectrum, respectively, are Borel maps, where $\mathcal{L}(X)(\operatorname{resp} . \mathcal{K}(\mathbb{C}))$ is endowed with the strong operator topology (resp. Hausdorff topology). This enables us to derive the topological complexity of some subsets of $\mathcal{L}(X)$ and to discuss the properties of a class of strongly continuous semigroups. We close the paper by giving a characterization of strongly continuous semigroups on hereditarily indecomposable Banach spaces.
\end{abstract}

1. Introduction. Let $X$ be an infinite-dimensional Banach space over the complex field and let $\mathcal{L}(X)$ denote the algebra of bounded linear operators on $X$. The subset of all finite rank (resp. compact) operators of $\mathcal{L}(X)$ is designated by $\mathcal{F}_{0}(X)$ (resp. $\mathcal{K}(X)$ ). One can consider on $\mathcal{L}(X)$ its strong topology which is the coarsest topology for which the maps $\varphi_{x}: \mathcal{L}(X) \rightarrow X$, $\varphi_{x}(T)=T x$, are continuous for all $x \in X$. Equipped with this topology, $\mathcal{L}(X)$ is a topological vector space denoted by $\mathcal{L}_{\mathrm{s}}(X)$. For $T \in \mathcal{L}(X)$, we let $\sigma(T), \varrho(T), N(A)$ and $R(T)$ denote the spectrum, resolvent set, null space and range of $T$, respectively. The nullity of $T, \alpha(T)$, is the dimension of $N(T)$, and the deficiency of $T, \beta(T)$, is the codimension of $R(T)$ in $X$. The set of upper semi-Fredholm operators of $\mathcal{L}(X)$ is defined by

$$
\Phi_{+}(X)=\{T \in \mathcal{L}(X): \alpha(T)<\infty \text { and } R(T) \text { is closed in } X\},
$$

and the set of lover semi-Fredholm operators of $\mathcal{L}(X)$ is defined by

$$
\Phi_{-}(X)=\{T \in \mathcal{L}(X): \beta(T)<\infty(\text { and } R(T) \text { is closed in } X)\} .
$$

2000 Mathematics Subject Classification: 54H05, 47A10, 47D03.

Key words and phrases: essential spectra, Borel maps, strictly singular operators, Fredholm operators, Riesz operators, strongly continuous semigroups, hereditarily indecomposable Banach spaces. 
Operators in $\Phi(X)=\Phi_{+}(X) \cap \Phi_{-}(X)$ are called Fredholm operators of $\mathcal{L}(X)$. If $T \in \Phi(X)$, the number $i(T)=\alpha(T)-\beta(T) \in \mathbb{Z}$ is called the index of $T$. The Fredholm domain of $T, \Phi_{T}$, is the set of all complex numbers $\lambda$ such that $\lambda I-T$ is a Fredholm operator. Finally, we denote by $\Phi_{0}(X)$ the set of Weyl operators on $X$, that is,

$$
\Phi_{0}(X)=\{T \in \Phi(X): i(T)=0\} .
$$

Let $S_{X}$ denote the unit sphere of $X$ and $T \in \mathcal{L}(X)$. The following definitions are well known: the approximate point spectrum of $T$ is

$$
\sigma_{\text {ap }}(T)=\left\{\lambda \in \mathbb{C}: \exists\left(x_{n}\right)_{n \in \mathbb{N}}, x_{n} \in S_{X} \text { and } \lim _{n \rightarrow \infty}\left\|(\lambda-T) x_{n}\right\|=0\right\},
$$

the compression spectrum of $T$ is

$$
\sigma_{\text {com }}(T)=\{\lambda \in \mathbb{C}:(\lambda-T) X \text { is not dense in } X\},
$$

the essential spectrum of $T$ (the spectrum of $T$ in the Calkin algebra) is

$$
\sigma_{\mathrm{e}}(T):=\{\lambda \in \mathbb{C}: \lambda-T \notin \Phi(X)\},
$$

the Weyl essential spectrum of $T$ is

$$
\sigma_{\mathrm{w}}(T)=\left\{\lambda \in \mathbb{C}: \lambda-T \notin \Phi_{0}(X)\right\},
$$

and the Browder essential spectrum is

$$
\sigma_{\mathrm{b}}(T)=\bigcap_{K \in \mathcal{K}(X)}\{\sigma(T+K): T K=K T\}
$$

Let $\pi_{0}(T)$ denote the set of isolated points of $\sigma(T)$ whose associated spectral projections have finite-dimensional ranges. It is well known that $\sigma_{\mathrm{b}}(T)=$ $\sigma(T) \backslash \pi_{0}(T)$.

In Chapter 3 of his thesis [24], Yahdi discussed the topological complexity of some subsets of $\mathcal{L}(X)$ under the hypotheses that $X$ is a separable Banach space and $\mathcal{L}(X)$ is endowed with the strong operator topology. In particular, he showed that the families of stable, ergodic and power bounded operators are Borel subsets of $\mathcal{L}(X)$ while the set of superstable operators is coanalytic. Other results in this direction may be found in Chapter 4 of Yahdi's work.

The purpose of this work is twofold: Firstly, we establish further results in the spirit of those obtained in [24] (cf. Theorem 1.1 below). Secondly, we apply our result to derive the topological complexity of some subsets of $\mathcal{L}(X)$ equipped with the strong operator topology (Corollary 1.1) and to discuss the properties of strongly continuous semigroups in Banach spaces under a hypothesis $(\mathcal{A})$ (cf. Section 4 ). To state our results, let us first introduce some notions and notations.

Let $X$ be a Banach space. An operator $T \in \mathcal{L}(X)$ is called a left topological divisor of zero (briefly, left TDZ) in $\mathcal{L}(X)$ if there exists a sequence $\left(x_{n}\right)_{n \in \mathbb{N}}$ of vectors such that $\left\|x_{n}\right\|=1$ and $T x_{n} \rightarrow 0$ as $n \rightarrow \infty$. Let $\mathcal{T}(X)$ denote the set of all operators of $\mathcal{L}(X)$ which are left TDZ. It is well known 
that the approximate point spectrum and $\mathcal{T}(X)$ are connected in the following way:

$$
\sigma_{\text {ap }}(T)=\{\lambda \in \mathbb{C}: \lambda I-T \in \mathcal{T}(X)\} .
$$

An operator $R \in \mathcal{L}(X)$ is called a Riesz operator if $\lambda I-R \in \Phi(X)$ for all scalars $\lambda \neq 0$, i.e. $\Phi_{R}=\mathbb{C} \backslash\{0\}$. Let $\mathcal{R}(X)$ denote the class of all Riesz operators. Finally, we denote by $\operatorname{Inv}(X)$ the subset of $\mathcal{L}(X)$ consisting of all invertible operators on $X$. For more information about the notions and concepts introduced above we refer, for example, to $[1,8,12,19,20]$.

Let $\mathbb{C}$ be the field of complex numbers and let $\mathcal{K}(\mathbb{C})$ be the collection of all nonempty compact subsets of $\mathbb{C}$. For $K$ and $K^{\prime}$ in $\mathcal{K}(\mathbb{C})$, we define the Hausdorff distance $d_{\mathrm{H}}\left(K, K^{\prime}\right)$ between $K$ and $K^{\prime}$ by

$$
d_{\mathrm{H}}\left(K, K^{\prime}\right)=\max \left(\delta\left(K, K^{\prime}\right), \delta\left(K^{\prime}, K\right)\right),
$$

where $\delta\left(K, K^{\prime}\right)=\sup _{x \in K} d\left(x, K^{\prime}\right)$. In the rest of this paper $\mathcal{K}(\mathbb{C})$ will be endowed with this metric.

We are now ready to state the main result of this paper.

Theorem 1.1. Let $X$ be a Banach space. If $X$ is separable, then the maps

(1) $\sigma_{\text {ap }}: \mathcal{L}_{\mathrm{s}}(X) \rightarrow \mathcal{K}(\mathbb{C}), T \mapsto \sigma_{\text {ap }}(T)$,

(2) $\sigma: \mathcal{L}_{\mathrm{S}}(X) \rightarrow \mathcal{K}(\mathbb{C}), T \mapsto \sigma(T)$,

are Borel maps. If further $X^{\prime}$ (the dual space of $X$ ) is separable, then

(3) $\sigma_{\mathrm{w}}: \mathcal{L}_{\mathrm{s}}(X) \rightarrow \mathcal{K}(\mathbb{C}), T \mapsto \sigma_{\mathrm{w}}(T)$,

(4) $\sigma_{\mathrm{e}}: \mathcal{L}_{\mathrm{S}}(X) \rightarrow \mathcal{K}(\mathbb{C}), T \mapsto \sigma_{\mathrm{e}}(T)$,

$(5) \sigma_{\mathrm{b}}: \mathcal{L}_{\mathrm{s}}(X) \rightarrow \mathcal{K}(\mathbb{C}), T \mapsto \sigma_{\mathrm{b}}(T)$,

are Borel maps.

Evidently, in the case where $\mathcal{L}(X)$ is endowed with the operator norm topology, the spectrum and essential spectrum are known to be upper semicontinuous (hence Borel) but, in general, not continuous.

REMARK 1.1. Let $\mathbb{T}$ denote the unit circle of the complex plane $\mathbb{C}$. Proposition IV.2.3 in Yahdi's thesis [24, p. 86] asserts that if $X$ is a separable Banach space, then the map $\sigma_{\mathbb{T}}: \mathcal{L}_{\mathrm{S}}(X) \rightarrow \mathcal{K}(\mathbb{T}), A \mapsto \sigma_{\mathbb{T}}(A)=\sigma(A) \cap \mathbb{T}$, is Borel. An examination of its proof shows that it contains implicitly the main steps of the proofs of items (1) and (2) of Theorem 1.1 where only minor changes are required. For completeness we will give the proof of these two statements. To our knowledge assertions (3)-(5) above are new.

As a consequence of Theorem 1.1 we have

Corollary 1.1. Let $X$ be a Banach space. If $X$ is separable, then $\mathcal{T}(X)$, $\operatorname{Inv}(X)$ and $\mathcal{R}(X)$ are Borel subsets of $\mathcal{L}_{\mathrm{s}}(X)$. If , further, $X^{\prime}$ is separable, then $\Phi(X)$ and $\Phi_{0}(X)$ are also Borel subsets of $\mathcal{L}_{\mathrm{s}}(X)$. 
Recall that if $T \in \mathcal{L}(X)$, the compression spectrum of $T$ may also be defined by

$$
\sigma_{\text {com }}(T)=\{\lambda \in \mathbb{C}: \lambda-T \text { is a right divisor of zero }\} .
$$

Let $\mathcal{Z}(X)$ denote the set of right divisors of zero in $\mathcal{L}(X)$ and $\mathcal{Z}^{\mathrm{c}}(X)=$ $\mathcal{L}(X) \backslash \mathcal{Z}(X)$. Clearly, we have

$$
\mathcal{Z}^{\mathrm{c}}(X)=\left\{T \in \mathcal{L}(X): 0 \notin \sigma_{\mathrm{com}}(T)\right\} .
$$

Corollary 1.1 asserts that if $X$ is a separable Banach space, then $\mathcal{T}(X)$ and $\operatorname{Inv}(X)$ are Borel subsets of $\mathcal{L}_{\mathrm{S}}(X)$, and if $X^{\prime}$ is separable, $\Phi_{0}(X)$ and $\Phi(X)$ enjoy the same property. The following proposition gives more details on the topological structure of these sets.

Proposition 1.1. If $X$ is a separable Banach space, then $\mathcal{T}(X)$ is a $G_{\delta}$ subset of $\mathcal{L}_{\mathrm{s}}(X), \mathcal{Z}(X)$ is an $F_{\sigma}$ subset of $\mathcal{L}_{\mathrm{s}}(X)$, and $\operatorname{Inv}(X)$ is a Borel subset of $\mathcal{L}_{\mathrm{S}}(X)$ of the form $F_{\sigma} \backslash F_{\sigma}$. If further $X^{\prime}$ is separable, then $\Phi_{0}(X)$ and $\Phi(X)$ are countable unions of Borel subsets of $\mathcal{L}_{\mathrm{S}}(X)$ of the form $F_{\sigma} \backslash F_{\sigma}$.

The remainder of this paper is organized as follows. In Section 2, we recall and gather some classical facts from functional analysis and descriptive set theory required later. The proofs of Theorem 1.1, Corollary 1.1 and Proposition 1.1 are the topic of Section 3. In Section 4 we present a characterization of strongly continuous semigroups $(T(t))_{t \geq 0}$ under the assumption that, for all $t>0, \sigma_{\mathrm{e}}(T(t))=\{\lambda(t)\}$ on Banach spaces with separable duals. Note that semigroups satisfying this condition were already discussed in $[2,10,13]$.

The case where $\sigma_{\mathrm{e}}(T(t))=\left\{\lambda_{1}(t), \ldots, \lambda_{n}(t)\right\}(n \geq 2)$ was considered in $[14,15]$ under the hypothesis that $\sigma_{\mathrm{e}}(T(t))$ depends continuously on $t$. In particular, it was proved that if $\lambda_{i}(t) \neq 0$ for all $t>0$ and $i=1, \ldots, n$, then $(T(t))_{t \geq 0}$ can be embedded in a uniformly continuous group. However, if $\lambda_{i}\left(t_{0}\right)=0$ for some $i \in\{1, \ldots, n\}$ and $t_{0}>0$, then $\lambda_{i}(t)=0$ for all $t>0$ and there is a spectral decomposition of $X$ of the form $X=X_{0} \oplus X_{1}$ where $\left(T(t)_{\mid X_{0}}\right)_{t \geq 0}$ is a strongly continuous semigroup of Riesz type on $X_{0}$, i.e. $T(t)_{\mid X_{0}} \in \mathcal{R}\left(X_{0}\right)$ for all $t>0$. Its generator $A_{\mid X_{0}}$ is unbounded and $\left(\lambda-A_{\mid X_{0}}\right)^{-1} \in \mathcal{R}\left(X_{0}\right)$ for any $\lambda \in \varrho\left(A_{\mid X_{0}}\right)$. Moreover, the part of $(T(t))_{t \geq 0}$ in $X_{1},\left(T(t)_{\mid X_{1}}\right)_{t \geq 0}$, can be embedded in a uniformly continuous group. In $[2,10,13]$ we have $n=1$ and $\lambda(\cdot)$ is assumed to be constant $(\lambda(t)=1$ for all $t>0$ ), thus continuous.

In our context, making use of Theorem 1.1, we prove that $\lambda(\cdot)$ is a Borel function from $[0, \infty)$ into $\mathbb{C}$, which relaxes the continuity hypothesis. Moreover, we show that either $(T(t))_{t \geq 0}$ is a semigroup of Riesz operators and $A$ is unbounded and $(\lambda-A)^{-1} \in \mathcal{R}(X)$ for each $\lambda \in \varrho\left(A_{\mid X_{0}}\right)$, or else $(T(t))_{t \geq 0}$ is embeddable in a uniformly continuous group and there exists $\beta \in \mathbb{C}$ such that $A-\beta I$ is a Riesz operator. 
In the last part of this section we prove that, on hereditarily indecomposable Banach spaces with separable duals (cf. [7, 4]), strongly continuous semigroups exhibit a nice behavior. In particular, we establish that the infinitesimal generator of any strongly continuous group is necessarily bounded (which seems to be a feature of this class of spaces).

2. Preliminaries. In this section we will collect some notions and tools from functional analysis and descriptive set theory required in the rest of the paper.

Proposition 2.1. Let $X$ be a Banach space and let $T \in \mathcal{L}(X)$.

(1) Except the compression spectrum, all the subsets of $\sigma(T)$ defined above are compact. In general, the compression spectrum does not have this property because the compression spectrum of the unilateral shift is the unit open disc (cf. [8, Problem 82]).

(2) If $\lambda$ is an isolated point of $\sigma(T)$, then either $\lambda \in \pi_{0}(T)$ or $\lambda \in \sigma_{\mathrm{w}}(T)$. (It suffices to consider the spectral decomposition of $T$ associated to $\{\lambda\}$ and $\sigma(T) \backslash\{\lambda\}$.)

(3) Let $\mathcal{F}_{0}(X)$ be the ideal of finite rank operators in $\mathcal{L}(X)$. Then

$$
\sigma_{\mathrm{w}}(T)=\bigcap_{F \in \mathcal{F}_{0}(X)} \sigma(T+F) .
$$

This result remains valid if we replace $\mathcal{F}_{0}(X)$ by any ideal of Riesz operators (compact, strictly singular, etc.; cf. [12]).

(4) If $X^{\prime}$ (the topological dual of $X$ ) is separable, then $\mathcal{F}_{0}(X)$ is separable for the norm topology induced by $\mathcal{L}(X)$. (It suffices to write $T \in$ $\mathcal{F}_{0}(X)$ as a finite sum of operators of the form $x^{\prime} \otimes x$ with $x \in X$, $x^{\prime} \in X^{\prime}$ and use the separability of both $X$ and $X^{\prime}$.)

Let $Z$ be a topological space. For any subset $A$ of $Z$, let

$$
d(A)=\{x \in A: x \text { is an accumulation point of } A\} .
$$

The map which assigns to each subset $A$ of $Z$ the set $d(A)$ is called the Cantor-Bendixson derivative. Obviously, $d(A)$ is closed.

Proposition 2.2.

(1) The topology of $\mathcal{K}(\mathbb{C})$ is generated by two families of open sets in $\mathcal{K}(\mathbb{C})$ :

$$
\mathcal{T}^{1}=\{K \in \mathcal{K}(\mathbb{C}): K \subset U\} \quad \text { and } \quad \mathcal{T}^{2}=\{K \in \mathcal{K}(\mathbb{C}): K \cap U \neq \emptyset\}
$$
for any open set $U$ in $\mathbb{C}$.

(2) The Borel structure of $\mathcal{K}(\mathbb{C})$ is generated by each of these two families.

(3) The Cantor-Bendixson derivative $d: \mathcal{K}(\mathbb{C}) \rightarrow \mathcal{K}(\mathbb{C})$ is a Borel map. 
A topological space is said to be Polish if it is separable and there is a complete metric determining its topology. Note that $\mathbb{R}, \mathbb{C}, \mathbb{R}^{n}$ and $\mathbb{C}^{n}$ are Polish spaces. For other examples and the properties of this class of spaces see, for example, [11]. For later use we recall that if a topological space $X$ is Polish, then $\mathcal{K}(X)$ is also Polish (see Theorem 4.25 in [11]). Accordingly, $\mathcal{K}(\mathbb{C})$ is a Polish space.

We say that a topological space is a standard Borel space if it is a topological space whose Borel structure (but not necessarily the topological structure) is the same as that of a Polish space. We will make use of the next result (cf. [11, p. 80]).

Proposition 2.3. If $X$ is a separable Banach space, then $\mathcal{L}_{\mathrm{s}}(X)$ is a standard Borel space.

We close this section by recalling the following result due to Saint Raymond [18].

Proposition 2.4. Let $X$ be a standard Borel space and $Y$ a Polish space. If $\Omega \subseteq X \times Y$ is a Borel set for the product structure and, for every $x \in X, C_{x}=\{y \in Y:(x, y) \in \Omega\}$ is a $K_{\sigma}$ set (a countable union of compact sets) in $Y$, then $P_{X}(\Omega)$ (the range of the first projection of $\Omega$ ) is a Borel set in $X$.

3. Proofs. The proof of Theorem 1.1 requires the following two lemmas.

Lemma 3.1. Let $\psi: \mathcal{L}_{\mathrm{s}}(X) \rightarrow \mathcal{K}(\mathbb{C})$. Then $\psi$ is a Borel map if and only if

$$
\triangle_{\psi}=\{(T, \lambda) \in \mathcal{L}(X) \times \mathbb{C}: \lambda \in \psi(T)\}
$$

is a Borel set in $\mathcal{L}_{\mathrm{s}}(X) \times \mathbb{C}$.

Proof. Let us first remark that $\triangle=\{(K, \lambda) \in \mathcal{K}(\mathbb{C}) \times \mathbb{C}: \lambda \in K\}$ is closed in $\mathcal{K}(\mathbb{C}) \times \mathbb{C}$. To see this, take $\left(K_{0}, \lambda_{0}\right) \notin \triangle$. Then there exist two open sets $U, V$ such that $K_{0} \subset U, \lambda_{0} \in V$ and $U \cap V=\emptyset$. Hence, $\{K \in \mathcal{K}(\mathbb{C}): K \subset U\} \times V$ is open in $\mathcal{K}(\mathbb{C}) \times \mathbb{C}$, contains $\left(K_{0}, \lambda_{0}\right)$ and does not intersect $\triangle$ (because $\bigcup\{K \in \mathcal{K}(\mathbb{C}): K \subset U\} \cap V=\emptyset)$. So, $(\mathcal{K}(\mathbb{C}) \times \mathbb{C}) \backslash \triangle$ is open and therefore $\triangle$ is closed.

Let $\psi: \mathcal{L}_{\mathrm{s}}(X) \rightarrow \mathcal{K}(\mathbb{C})$ be a Borel map. Then $\psi \times I: \mathcal{L}_{\mathrm{S}}(X) \times \mathbb{C} \rightarrow$ $\mathcal{K}(\mathbb{C}) \times \mathbb{C},(\psi \times I)(T, \lambda)=(\psi(T), \lambda)$, is also a Borel map and we have $\triangle_{\psi}=(\psi \times I)^{-1}(\triangle)$. Accordingly, $\psi$ being Borel implies $\triangle_{\psi}$ is a Borel set.

To prove the converse, it is sufficient, by Proposition 2.2(2), to show that $\psi^{-1}(\{K \in \mathcal{K}(\mathbb{C}): K \cap U \neq \emptyset\})$ is a Borel set in $\mathcal{L}_{\mathrm{s}}(X)$ for every open set $U$ in $\mathbb{C}$. Let $P_{\mathcal{L}_{\mathrm{s}}(X)}: \mathcal{L}_{\mathrm{s}}(X) \times U \rightarrow \mathcal{L}_{\mathrm{s}}(X)$ be the projection and let $\Omega_{U, \psi}:=\{(T, \lambda) \in \mathcal{L}(X) \times U: \lambda \in \psi(T)\}$. Note that

$$
\begin{aligned}
& \{T \in \mathcal{L}(X): \psi(T) \cap U \neq \emptyset\} \\
& \quad=\{T \in \mathcal{L}(X): \exists \lambda \in U \text { such that } \lambda \in \psi(T)\}=P_{\mathcal{L}(X)}\left(\Omega_{U, \psi}\right) .
\end{aligned}
$$


For every $T \in \mathcal{L}(X)$,

$$
\begin{aligned}
C_{T}\left(\Omega_{U, \psi}\right): & =\left\{\lambda \in U:(T, \lambda) \in \Omega_{U, \psi}\right\}=\{\lambda \in U: \lambda \in \psi(T)\} \\
& =\psi(T) \cap U .
\end{aligned}
$$

Since every open set in $\mathbb{C}$ is a $K_{\sigma}$ set, so is $C_{T}\left(\Omega_{U, \psi}\right)$. On the other hand, for every open set $U$ in $\mathbb{C}$, we have $\Omega_{U, \psi}=\triangle_{\psi} \cap\left(\mathcal{L}_{\mathrm{s}}(X) \times U\right)$. Since $\triangle_{\psi}$ is a Borel set (by hypothesis) and $\mathcal{L}_{\mathrm{s}}(X) \times U$ is open, it follows that $\Omega_{U, \psi}$ is Borel. Now the use of Proposition 2.4 finishes the proof.

Let $n \in \mathbb{Z}$ and set

$$
\Phi_{n}(X)=\{U \in \Phi(X): \operatorname{ind}(U)=n\} .
$$

Lemma 3.2. Let $A \in \mathcal{L}(X)$ be a fixed operator satisfying $\operatorname{ind}(A)=-n$ where $n \in \mathbb{Z}$. Then

$$
\Phi_{n}(X)=\left\{T \in \mathcal{L}(X): A T \in \Phi_{0}(X)\right\}
$$

Proof. To see this, first observe that, by the Atkinson theorem, we have $\Phi_{n}(X) \subseteq\left\{T \in \mathcal{L}(X): A T \in \Phi_{0}(X)\right\}$. Now let $T \in \mathcal{L}(X)$ be such that $A T \in$ $\Phi_{0}(T)$ and consider $B \in \mathcal{L}(X)$ such that $B A=I+F$ where $F \in \mathcal{F}_{0}(X)$ with $\operatorname{ind}(B)=-\operatorname{ind}(A)=n$ and $B A T=T+F T$ ( $B$ exists because $A \in \Phi(X))$. Since $A T \in \Phi_{0}(X)$ and $B \in \Phi(X)$, we have $T \in \Phi(X)$ and

$$
\operatorname{ind}(T)=\operatorname{ind}(B A T)=\operatorname{ind}(B)+\operatorname{ind}(A T)=n .
$$

This shows that $T \in \Phi_{n}(X)$.

We are finally prepared for

Proof of Theorem 1.1. (1) By definition,

$$
\begin{aligned}
\sigma_{\text {ap }}(T) & =\left\{\lambda \in \mathbb{C}: \exists\left(x_{n}\right) \subset S_{X} \text { with } \lim _{n \rightarrow \infty}\left\|(\lambda-T) x_{n}\right\|=0\right\} \\
& =\left\{\lambda \in \mathbb{C}: \forall n \in \mathbb{N}, \exists x \in S_{X} \text { such that }\|(\lambda-T) x\|<1 / n\right\} .
\end{aligned}
$$

Let $\mathcal{D}$ be a countable dense subset of $S_{X}$ (which exists by separability). Clearly, there exists $x \in S_{X}$ such that $\|(\lambda-T) x\|<1 / n$ if and only if there exists $x \in \mathcal{D}$ such that $\|(\lambda-T) x\|<1 / n$. This leads to

$$
\sigma_{\text {ap }}(T)=\{\lambda \in \mathbb{C}: \forall n \in \mathbb{N}, \exists x \in \mathcal{D} \text { such that }\|(\lambda-T) x\|<1 / n\} .
$$

Following the notations of Lemma 3.1 we may write

$$
\begin{aligned}
\Delta_{\sigma_{\mathrm{ap}}} & =\left\{(T, \lambda) \in \mathcal{L}(X) \times \mathbb{C}: \lambda \in \sigma_{\mathrm{ap}}(T)\right\} \\
& =\{(T, \lambda) \in \mathcal{L}(X) \times \mathbb{C}: \forall n \in \mathbb{N}, \exists x \in \mathcal{D} \text { such that }\|(\lambda-T) x\|<1 / n\} \\
& =\bigcap_{n \in \mathbb{N}} \bigcup_{x \in \mathcal{D}} A_{x, n}
\end{aligned}
$$

where $A_{x, n}=\{(T, \lambda) \in \mathcal{L}(X) \times \mathbb{C}:\|(\lambda-T) x\|<1 / n\}$. By the definition of the strong operator topology, $(T, \lambda) \mapsto(\lambda-T) x$ is continuous from $\mathcal{L}_{\mathrm{s}}(X) \times \mathbb{C}$ 
into $X$. Thus $A_{x, n}$ is an open set in $\mathcal{L}_{\mathrm{S}}(X) \times \mathbb{C}$ (for every $x \in \mathcal{D}, n \in \mathbb{N}$ ) and therefore $\Delta_{\sigma_{\mathrm{ap}}}$ is a Borel set in $\mathcal{L}_{\mathrm{s}}(X) \times \mathbb{C}$. Now the result follows from Lemma 3.1.

(2) First recall that $\sigma(T)=\sigma_{\mathrm{ap}}(T) \cup \sigma_{\mathrm{com}}(T)$. So, $\Delta_{\sigma}=\Delta_{\sigma_{\mathrm{ap}}} \cup C$, where

$$
C=\left\{(T, \lambda) \in \mathcal{L}(X) \times \mathbb{C}: \lambda \in \sigma_{\operatorname{com}}(T)\right\} .
$$

Hence, in order to prove that the map $T \mapsto \sigma(T)$ is Borel, it suffices to establish that $C$ is a Borel set. To do so, observe that, by definition,

$$
\begin{aligned}
& C=\{(T, \lambda) \in \mathcal{L}(X) \times \mathbb{C}:(T-\lambda) X \text { is not dense in } X\} \\
&=\{(T, \lambda) \in \mathcal{L}(X) \times \mathbb{C}: \exists n \in \mathbb{N} \text { and } y \in X \text { with } \\
&\qquad \quad\|y-(T-\lambda) x\|>1 / n \text { for all } x \in X\} .
\end{aligned}
$$

Let $\mathcal{D}$ be a countable dense subset of $X$. We can see that

$$
\begin{aligned}
C=\{(T, \lambda) \in \mathcal{L}(X) \times \mathbb{C}: \exists n \in \mathbb{N} \text { and } y \in \mathcal{D} \text { with } \\
\qquad\|y-(T-\lambda) x\|>1 / n \text { for all } x \in \mathcal{D}\} .
\end{aligned}
$$

Hence, we can write

$$
C=\bigcup_{n \in \mathbb{N}} \bigcup_{y \in \mathcal{D}} \bigcap_{x \in \mathcal{D}}\{(T, \lambda) \in \mathcal{L}(X) \times \mathbb{C}:\|y-(T-\lambda) x\|>1 / n\} .
$$

As in (1), using the continuity for the strong operator topology, we conclude that $C$ is a Borel set. The result follows from Lemma 3.1.

(3) Let $\mathcal{D}$ be a countable dense subset of $\mathcal{F}_{0}(X)$ (the set of finite rank operators on $X$ with its norm topology). One can easily see that

$$
\sigma_{\mathrm{w}}(T)=\bigcap_{F \in \mathcal{F}_{0}(X)} \sigma(T+F)=\bigcap_{F \in \mathcal{D}} \sigma(T+F)
$$

and

$$
\begin{aligned}
\Delta_{\sigma_{\mathrm{w}}} & =\left\{(T, \lambda) \in \mathcal{L}(X) \times \mathbb{C}: \lambda \in \sigma_{\mathrm{w}}(T)\right\} \\
& =\left\{(T, \lambda) \in \mathcal{L}(X) \times \mathbb{C}: \lambda \in \bigcap_{F \in \mathcal{D}} \sigma(T+F)\right\} \\
& =\bigcap_{F \in \mathcal{D}}\{(T, \lambda) \in \mathcal{L}(X) \times \mathbb{C}: \lambda \in \sigma(T+F)\} \\
& =\bigcap_{F \in \mathcal{D}}\left\{\left(T^{\prime}-F, \lambda\right) \in \mathcal{L}(X) \times \mathbb{C}: \lambda \in \sigma\left(T^{\prime}\right)\right\}
\end{aligned}
$$

where $T^{\prime}=T+F$. So,

$$
\Delta_{\sigma_{\mathrm{w}}}=\bigcap_{F \in \mathcal{D}}\left(\Delta_{\sigma}-(F, 0)\right) .
$$


Clearly, for every $F \in \mathcal{D}$, the translation by $(-F, 0)$ of the Borel set $\Delta_{\sigma}$ is a Borel set in $\mathcal{L}_{\mathrm{s}}(X) \times \mathbb{C}$. Consequently, $\Delta_{\sigma_{\mathrm{w}}}$ is a Borel set and therefore $\sigma_{\mathrm{w}}(\cdot)$ is a Borel map.

(4) Let $A \in \mathcal{L}(X)$ and set $\sigma^{A}(T)=\{\lambda \in \mathbb{C}: \lambda A-T$ is not invertible $\}$. In general $\sigma^{A}(T)$ is not compact but only closed. Let $q \in \mathbb{N}$, and set $\sigma_{q}^{A}(T):=$ $\sigma^{A}(T) \cap B(0, q)$ (where $B(0, q)=\{\lambda \in \mathbb{C}:|\lambda| \leq q\}$ ). Then $T \mapsto \sigma_{q}^{A}(T)$ defines a map from $\mathcal{L}(X)$ into $\mathcal{K}(\mathbb{C})$. Note that

$$
\begin{aligned}
\Delta_{\sigma_{q}^{A}(T)}= & \left\{(T, \lambda) \in \mathcal{L}(X) \times \mathbb{C}: \lambda \in \sigma_{q}^{A}(T)\right\} \\
= & \left\{(T, \lambda) \in \mathcal{L}(X) \times \mathbb{C}:|\lambda| \leq q \text { and } \exists\left(x_{k}\right) \subseteq S_{X}\right. \text { with } \\
& \cup\left\{(T, \lambda) \in \mathcal{L}(X) \times \mathbb{C}:|\lambda| \leq q \text { and } \overline{\left.\lim _{k \rightarrow \infty}\left\|(\lambda A-T) x_{k}\right\|=0\right\}}\right. \\
= & \left\{(T, \lambda) \in \mathcal{L}(X) \times B(0, q): \forall r \in \mathbb{N}, \exists x \in S_{X}\right. \text { such that } \\
& \quad\|(\lambda A-T) x\|<1 / r\} \\
& \cup\{(T, \lambda) \in \mathcal{L}(X) \times B(0, q): \exists y \in X, \exists r \in \mathbb{N} \text { such that } \forall x \in X, \\
& \|y-(\lambda A-T) x\| \geq 1 / r\} .
\end{aligned}
$$

Note that

$\Omega_{1}:=\left\{(T, \lambda) \in \mathcal{L}(X) \times B(0, q): \forall r \in \mathbb{N}, \exists x \in S_{X}\right.$ such that

$$
\begin{aligned}
& \|(\lambda A-T) x\|<1 / r\} \\
= & \left\{(T, \lambda) \in \mathcal{L}(X) \times \mathbb{C}: \forall r \in \mathbb{N}, \exists x \in S_{X} \text { such that }\|(\lambda A-T) x\|<1 / r\right\} \\
& \cap(\mathcal{L}(X) \times B(0, q)) \\
= & \left(\bigcap_{r \in \mathbb{N}} \bigcup_{x \in X} O_{A, x, r}\right) \cap(\mathcal{L}(X) \times B(0, q)),
\end{aligned}
$$

where

$$
O_{A, x, r}=\{(T, \lambda) \in \mathcal{L}(X) \times \mathbb{C}:\|(\lambda A-T) x\|<1 / r\},
$$

which is an open subset of $\mathcal{L}_{\mathrm{s}}(X) \times \mathbb{C}$. Hence $\Omega_{1}$ is a Borel set.

Similarly, $\Omega_{2}:=\{(T, \lambda) \in \mathcal{L}(X) \times B(0, q): \forall y \in \mathcal{D}, \exists r \in \mathbb{N}$ such that $\forall x \in X$,

$$
\left.=\left(\bigcap_{y \in \mathcal{D}} \bigcup_{r \in \mathbb{N}} \bigcap_{x \in X} F_{A, x, y, r}\right) \cap\left(\mathcal{L}_{\mathrm{s}}(X) \times B(0, q)\right), \quad\|y-(\lambda A-T) x\| \geq 1 / r\right\}
$$

where

$$
F_{A, x, y, r}=\{(T, \lambda) \in \mathcal{L}(X) \times \mathbb{C}:\|(\lambda A-T) x-y\| \geq 1 / r\}
$$

is a closed subset of $\mathcal{L}_{\mathrm{S}}(X) \times \mathbb{C}$ and $\mathcal{D}$ is a countable dense subset of $X$. So, $\Omega_{2}$ is then a Borel set of $\mathcal{L}_{\mathrm{s}}(X) \times \mathbb{C}$. Accordingly, $\Delta_{\sigma_{q}^{A}}=\Omega_{1} \cup \Omega_{2}$ is a Borel 
set. Hence, for all $q \in \mathbb{N}$ and $A \in \mathcal{L}_{\mathrm{S}}(X)$ fixed, $T \mapsto \sigma_{q}^{A}(T)$ is a Borel map from $\mathcal{L}_{\mathrm{s}}(X)$ into $\mathcal{K}(\mathbb{C})$. Set

$$
\sigma_{0, q}^{A}(T)=\left\{\lambda \in \mathbb{C}: \lambda A-T \notin \Phi_{0}(X)\right\} \cap B(0, q)
$$

$\left(\sigma_{0, q}^{A}(T)\right.$ is a compact subset of $\left.\mathbb{C}\right)$. Then

$$
\sigma_{0, q}^{A}(T)=\bigcap_{F \in \mathcal{F}_{0}(X)} \sigma_{q}^{A}(T+F)=\bigcap_{F \in \mathcal{F}_{0}^{d}(X)} \sigma_{q}^{A}(T+F),
$$

where $\mathcal{F}_{0}^{d}(X)$ is a countable dense subset of $\mathcal{F}_{0}(X)$. So, $T \mapsto \sigma_{0, q}^{A}(T)$ is a Borel map.

Recall that $\sigma_{\mathrm{e}}(T)=\mathbb{C} \backslash \Phi_{T}$ and $\Phi(X)=\bigcup_{k \in \mathbb{Z}} \Phi_{k}(X)$ (for some $k \in \mathbb{Z}$, $\Phi_{k}(X)$ may be empty). Then

$$
\sigma_{\mathrm{e}}(T)=\bigcap_{k \in \mathbb{Z}}\left\{\lambda \in \mathbb{C}: \lambda-T \notin \Phi_{k}(X)\right\} .
$$

Making use of Lemma 3.2 we may write

$$
\sigma_{\mathrm{e}}(T)=\bigcap_{k \in \mathbb{Z}}\left\{\lambda \in \mathbb{C}: A_{k}(\lambda-T) \notin \Phi_{0}(X)\right\},
$$

where, for all $k, A_{k}$ is a fixed operator with $\operatorname{ind}\left(A_{k}\right)=-k$. Hence,

$$
\begin{aligned}
\sigma_{\mathrm{e}}(T) \cap B(0, q) & =\bigcap_{k \in \mathbb{Z}}\left(\left\{\lambda \in \mathbb{C}: \lambda A_{k}-A_{k} T \notin \Phi_{0}(X)\right\} \cap B(0, q)\right) \\
& =\bigcap_{k \in \mathbb{Z}} \sigma_{0, q}^{A_{k}}\left(A_{k} T\right) .
\end{aligned}
$$

Put

$$
\sigma_{\mathrm{e}, q}(T)=\sigma_{\mathrm{e}}(T) \cap B(0, q)
$$

Then

$$
\sigma_{\mathrm{e}, q}(T)=\bigcap_{k \in \mathbb{Z}} \sigma_{0, q}^{A_{k}}\left(A_{k} T\right) .
$$

Since the map $T \mapsto A_{k} T$ is continuous from $\mathcal{L}_{\mathrm{s}}(X)$ into $\mathcal{L}_{\mathrm{s}}(X)$ and $T \mapsto$ $\sigma_{0, q}^{A_{k}}(T)$ defines a Borel map for all $q$ and $k$ in $\mathbb{N}$, it follows that $T \mapsto \sigma_{\mathrm{e}, q}(T)$ is a Borel map. This shows that $\Delta_{\sigma_{\mathrm{e}}}=\bigcup_{q \in \mathbb{N}} \Delta_{\sigma_{\mathrm{e}, q}}$, which is Borel. Now the use of Lemma 3.1 gives the result.

(5) First observe that, by Proposition 2.1(2), we may write $\sigma_{\mathrm{b}}(T)=$ $\sigma_{\mathrm{e}}(T) \cup d(\sigma(T))$ where $d$ is the Cantor-Bendixson derivative. Hence,

$$
\Delta_{\sigma_{\mathrm{b}}}=\Delta_{\sigma_{\mathrm{e}}} \cup \Delta_{d \circ \sigma} .
$$

We know, by item (4) of the theorem, that $\Delta_{\sigma_{\mathrm{e}}}$ is a Borel set. On the other hand, applying Lemma 3.1 and Proposition 2.2(3) one sees that $\Delta_{d \circ \sigma}$ is a Borel set in $\mathcal{L}_{\mathrm{s}}(X) \times \mathbb{C}$, so $\Delta_{\sigma_{\mathrm{b}}}$ is Borel. This gives the desired result and completes the proof of the theorem. 
Proof of Corollary 1.1. Consider the set $\mathcal{T}(X)$. Since the set of Borel sets is closed under complementation, it suffices to prove that $\mathcal{T}^{\mathrm{c}}(X)$, the complement of $\mathcal{T}(X)$ in $\mathcal{L}_{\mathrm{s}}(X)$, is Borel. To do so, let us observe that $\mathcal{T}^{\mathrm{c}}(X)=\left\{T \in \mathcal{L}(X): 0 \notin \sigma_{\text {ap }}(T)\right\}$. This may be written as

$$
\mathcal{T}^{\mathrm{c}}(X)=\sigma_{\text {ap }}^{-1}(\{K \in \mathcal{K}(\mathbb{C}): 0 \notin K\}) .
$$

But the set $\{K \in \mathcal{K}(\mathbb{C}): 0 \notin K\}$ is open in $\mathcal{K}(\mathbb{C})$, so applying Theorem 1.1(1) one concludes that $\mathcal{T}^{\mathrm{c}}(X)$ is Borel. Similarly, the sets $\operatorname{Inv}(X)$, $\Phi(X)$ and $\Phi_{0}(X)$ may be written, respectively, in the form

$$
\begin{aligned}
\operatorname{Inv}(X) & =\{T \in \mathcal{L}(X): 0 \notin \sigma(T)\}=\sigma^{-1}(\{K \in \mathcal{K}(\mathbb{C}): 0 \notin K\}), \\
\Phi(X) & =\left\{T \in \mathcal{L}(X): 0 \notin \sigma_{\mathrm{e}}(T)\right\}=\sigma_{\mathrm{e}}^{-1}(\{K \in \mathcal{K}(\mathbb{C}): 0 \notin K\}), \\
\Phi_{0}(X) & =\left\{T \in \mathcal{L}(X): 0 \notin \sigma_{\mathrm{w}}(T)\right\}=\sigma_{\mathrm{w}}^{-1}(\{K \in \mathcal{K}(\mathbb{C}): 0 \notin K\}) .
\end{aligned}
$$

Arguing as above and using statements (2)-(4) of Theorem 1.1 we infer that $\operatorname{Inv}(X), \Phi(X)$ and $\Phi_{0}(X)$ are Borel subsets of $\mathcal{L}_{\mathrm{s}}(X)$.

Next to prove that $\mathcal{R}(X)$ is a Borel subset of $\mathcal{L}_{\mathrm{S}}(X)$ it suffices to observe that $\mathcal{R}(X)=(\sigma \circ d)^{-1}(\{0\})$ where $d$ is the Cantor-Bendixson derivative and invoke the Borel character of the functions $\sigma(\cdot)$ and $d(\cdot)$ (cf. Theorem 1.1(2) and Proposition 2.2(3)).

Proof of Proposition 1.1. Let $D_{X}$ denote a countable dense subset of $X$. If $T \in \mathcal{L}(X)$, then $0 \notin \sigma_{\text {com }}(T)$ is equivalent to the fact that for all $n \in \mathbb{N}^{*}$ and $x \in D_{X}$ there is $y \in X$ such that $\|T y-x\|<1 / n$. So,

$$
\left\{T \in \mathcal{L}(X): 0 \notin \sigma_{\mathrm{com}}(T)\right\}=\bigcap_{n \in \mathbb{N}^{*}} \bigcap_{x \in D_{X}} \bigcup_{y \in X}\{T \in \mathcal{L}(X):\|T y-x\|<1 / n\} .
$$

This shows that $\mathcal{Z}^{\mathrm{c}}(X)$ is a countable intersection of open subsets of $\mathcal{L}_{\mathrm{s}}(X)$. Therefore $\mathcal{Z}^{\mathrm{c}}(X)$ is a $G_{\delta}$ subset of $\mathcal{L}_{\mathrm{s}}(X)$, which proves the assertion for $\mathcal{Z}(X)$.

We have already mentioned in the proof of Theorem 1.1(1) that the set $\mathcal{T}^{\mathrm{c}}(X)$ equals $\left\{T \in \mathcal{L}(X): 0 \notin \sigma_{\text {ap }}(T)\right\}$. Let $S_{X}$ denote the unit sphere of $X$. It is not difficult to see that $T \in\left\{U \in \mathcal{L}(X): 0 \notin \sigma_{\text {ap }}(U)\right\}$ if and only if $T \in \bigcup_{n \in \mathbb{N}^{*}}\left\{U \in \mathcal{L}(X): \forall x \in S_{X}\|U x\| \geq 1 / n\right\}$ or equivalently

$$
T \in \bigcup_{n \in \mathbb{N}^{*}} \bigcap_{x \in S_{X}}\{U \in \mathcal{L}(X):\|U x\| \geq 1 / n\} .
$$

Accordingly, $\mathcal{T}^{\mathrm{c}}(X)$ is a countable union of closed sets, that is, an $F_{\sigma}$ subset of $\mathcal{L}_{\mathrm{s}}(X)$. This proves the statement for $\mathcal{T}(X)$.

Now observe that

$$
\operatorname{Inv}(X)=\left\{T \in \mathcal{L}(X): 0 \notin \sigma_{\mathrm{ap}}(T)\right\} \cap\left\{T \in \mathcal{L}(X): 0 \notin \sigma_{\mathrm{com}}(T)\right\} .
$$

Consequently, $\operatorname{Inv}(X)=\mathcal{T}^{\mathrm{c}}(X) \cap \mathcal{Z}^{\mathrm{c}}(X)=\mathcal{T}^{\mathrm{c}}(X) \backslash \mathcal{Z}(X)$, which gives the desired result. 
Consider now the set of Weyl operators. It is not difficult to see that $T \in \Phi_{0}(X)$ if and only if $0 \notin \sigma_{\mathrm{w}}(T)$ if and only if $0 \notin \bigcap_{F \in \mathcal{F}_{0}(X)} \sigma(T+F)$. So,

$$
\Phi_{0}(X)=\left\{T \in \mathcal{L}(X): 0 \notin \bigcap_{F \in \mathcal{F}_{0}(X)} \sigma(T+F)\right\} .
$$

Let $\mathcal{D}$ be a countable dense subset of $\mathcal{F}_{0}(X)$. Arguing as in the proof of Theorem 1.1(3) one sees that

$$
\begin{aligned}
\Phi_{0}(X) & =\left\{T \in \mathcal{L}(X): 0 \notin \bigcap_{F \in \mathcal{D}} \sigma(T+F)\right\} \\
& =\left\{T \in \mathcal{L}(X): \exists F_{n} \in \mathcal{D} \text { such that } 0 \in \varrho\left(T+F_{n}\right)\right\} \\
& =\left\{T \in \mathcal{L}(X): \exists F_{n} \in \mathcal{D} \text { such that } T+F_{n} \text { is invertible }\right\} \\
& =\bigcup_{F \in \mathcal{D}}\{T \in \mathcal{L}(X): T+F \in \operatorname{Inv}(X)\} .
\end{aligned}
$$

Denote by $\mathcal{T}_{F}$ the translation from $\mathcal{L}(X)$ into itself which assigns to each $T$ the operator $T+F$. Then we may write $\Phi_{0}(X)=\bigcup_{F \in \mathcal{D}} \mathcal{T}_{F}^{-1}(\operatorname{Inv}(X))$. Now using the continuity of $\mathcal{T}_{F}$ and the fact that $\operatorname{Inv}(X)=\mathcal{T}^{\mathrm{c}}(X) \backslash \mathcal{Z}(X)$, we get

$$
\Phi_{0}(X)=\bigcup_{F \in \mathcal{D}} \mathcal{T}_{F}^{-1}\left(\mathcal{T}^{\mathrm{c}}(X)\right) \backslash \mathcal{T}_{F}^{-1}(\mathcal{Z}(X)),
$$

which ends the proof for $\Phi_{0}(X)$.

When dealing with the set of Fredholm operators one can write $\Phi(X)=$ $\bigcup_{n \in \mathbb{Z}} \Phi_{n}(X)$ where $\Phi_{n}(X)=\{T \in \Phi(X): i(T)=n\}$. According to Lemma 3.2, $\Phi_{n}(X)=\left\{T \in \mathcal{L}(X): A_{n} T \in \Phi_{0}(X)\right\}$ for each $n \in \mathbb{Z}$, where $A_{n}$ is a fixed operator satisfying $i(A)=-n$. Let $\mathcal{C}_{A}$ (with $A \in \mathcal{L}(X)$ ) denote the map from $\mathcal{L}(X)$ into itself defined by $\mathcal{C}_{A}(T)=A T$. Using the continuity of $\mathcal{C}_{A}$ and the fact that $\Phi_{n}(X)=\mathcal{C}_{A_{n}}^{-1}\left(\Phi_{0}(X)\right)$ for each $n \in \mathbb{Z}$, we infer that

$$
\Phi(X)=\bigcup_{n \in \mathbb{Z}} \mathcal{C}_{A_{n}}^{-1}\left(\Phi_{0}(X)\right),
$$

which completes the proof.

\section{Application to strongly continuous semigroups of operators.}

In this section we are interested in strongly continuous semigroups $(T(t))_{t \geq 0}$ defined on complex infinite-dimensional Banach spaces $X$ satisfying the condition

$$
\sigma_{\mathrm{e}}(T(t))=\{\lambda(t)\} \quad \text { for all } t>0 .
$$

4.1. Arbitrary Banach spaces. Let $X$ be a complex infinite-dimensional Banach space and let $(T(t))_{t \geq 0}$ be a strongly continuous semigroup on $X$ which satisfies $(\mathcal{A})$. Clearly, if $t, t^{\prime} \in[0, \infty)$, since $T(t)$ and $T\left(t^{\prime}\right)$ commute, 
we have $\sigma_{\mathrm{e}}\left(T(t) T\left(t^{\prime}\right)\right) \subseteq \sigma_{\mathrm{e}}(T(t)) \sigma_{\mathrm{e}}\left(T\left(t^{\prime}\right)\right)$. This leads to

$$
\lambda\left(t+t^{\prime}\right)=\lambda(t) \lambda\left(t^{\prime}\right) .
$$

In the following we denote by $\lambda(\cdot)$ the map from $[0, \infty)$ into $\mathbb{C}$ which assigns to each $t \in[0, \infty)$ the value $\lambda(t) \in \sigma_{\mathrm{e}}(T(t))$.

LEMma 4.1. If $X^{\prime}$ is separable and hypothesis $(\mathcal{A})$ holds true, then $\lambda(\cdot)$ is a Borel map.

REMARK 4.1. The separability hypothesis on $X^{\prime}$ is required to guarantee the Borel character of the function $T \mapsto \sigma_{\mathrm{e}}(T)$ from $\mathcal{L}_{\mathrm{s}}(X)$ into $\mathcal{K}(\mathbb{C})$ (cf. Theorem 1.1(4)).

Proof of Lemma 4.1. If $U$ is an open set of $\mathbb{C}$, then $\lambda^{-1}(U)=\{t \in[0, \infty)$ : $\{\lambda(t)\} \subset U\}$. Since $t \mapsto T(t)$ is continuous from $[0, \infty)$ into $\mathcal{L}_{\mathrm{s}}(X)$ and $T(t) \mapsto \sigma_{\mathrm{e}}(T(t))$ is a Borel map from $\mathcal{L}_{\mathrm{s}}(X)$ into $\mathcal{K}(\mathbb{C})$, by composition the map $t \mapsto\{\lambda(t)\}$ is Borel from $[0, \infty)$ into $\mathcal{K}(\mathbb{C})$. Note that $\lambda^{-1}(U)$ is the inverse image under $t \mapsto\{\lambda(t)\}$ of the set $\{K \in \mathcal{K}(\mathbb{C}): K \subset U\}$. But this set is open in $\mathcal{K}(\mathbb{C})$, therefore $\lambda^{-1}(U)$ is a Borel subset of $[0, \infty)$.

Lemma 4.2. Assume that hypothesis $(\mathcal{A})$ is satisfied. Then only one of the following two statements holds:

(1) There exists $t_{0}>0$ such that $\lambda\left(t_{0}\right)=0$ (and therefore $\lambda(t)=0$ for all $t \in] 0, \infty[)$.

(2) There exists $\alpha \in \mathbb{C}$ such that $\lambda(t)=e^{\alpha t}$ for all $t \in[0, \infty)$.

Proof. (1) Let $t_{0}>0$ be such that $\lambda\left(t_{0}\right)=0$. Using (4.1) one sees that $\left[\lambda\left(t_{0} / n\right)\right]^{n}=\lambda\left(t_{0}\right)=0$ for every $n \in \mathbb{N} \backslash\{0\}$. Hence $\lambda\left(t_{0} / n\right)=0$. Now let $t \in] 0, \infty\left[\right.$. Then there exists $n \in \mathbb{N} \backslash\{0\}$ such that $t_{0} / n \leq t$. Hence $\lambda(t)=\lambda\left(t_{0} / n\right) \cdot \lambda\left(t-t_{0} / n\right)=0$. Consequently, $\lambda(\cdot) \equiv 0$ on $] 0, \infty[$.

(2) It is well known that any nontrivial Borel solution of the functional equation (4.1) can be written in the form $\lambda(t)=e^{\alpha t}$ for some $\alpha \in \mathbb{C}$. So, by the preceding assertion, it suffices that there exists a real $t_{0}>0$ such that $\lambda\left(t_{0}\right) \neq 0$ to get $\lambda(t) \neq 0$ for all $\left.t \in\right] 0, \infty[)$.

REMARK 4.2. Actually, the second item of Lemma 4.2 remains valid if we assume only the measurability of $\lambda(\cdot)$ (see $[9$, pp. 144-145]). Hence, for such $\lambda(\cdot)$, measurability implies continuity and $\lambda(\cdot)$ admits an obvious extension to $\mathbb{R}$.

Now we will prove that the possibilities in Lemma 4.2 for the function $\lambda(\cdot)$ give rise to a dichotomy for the corresponding semigroup. To do so, first recall that for Riesz operators the sets $\sigma_{\mathrm{e}}(\cdot), \sigma_{\mathrm{w}}(\cdot)$, and $\sigma_{\mathrm{b}}(\cdot)$ coincide and reduce to $\{0\}$, and a semigroup $(T(t))_{t \geq 0}$ is said to be of Riesz type if $T(t)$ is a Riesz operator for all $t \in] 0, \infty[$. 
Proposition 4.1. Let $(T(t))_{t \geq 0}$ be a strongly continuous semigroup on a Banach space $X$ with infinitesimal generator $A$. Assume that $X^{\prime}$ is separable and hypothesis $(\mathcal{A})$ is satisfied. Then there are two alternatives: either $(T(t))_{t \geq 0}$ is a semigroup of Riesz type and $A$ is unbounded with nonempty resolvent set and with resolvent of Riesz type, or else $(T(t))_{t \geq 0}$ is embeddable in a uniformly continuous group and there exists $\beta \in \mathbb{C}$ such that $A-\beta I$ is a Riesz operator.

Note that the first alternative corresponds to the case $\lambda(t)=0$ for all $t>0$ and the second one concerns the case $\lambda(t) \neq 0$ for some (and hence for all) $t>0$. Before establishing the proposition we recall the following result proved in [3, p. 276].

Lemma 4.3. Let $(T(t))_{t \geq 0}$ be a strongly continuous semigroup with generator $A$ on a Banach space $X$. The following spectral inclusion holds:

$$
e^{t \sigma(A)} \subseteq \sigma(T(t)) \quad \text { for } t \geq 0 .
$$

Moreover, if $e^{\lambda_{0} t}$ is an isolated eigenvalue of $T(t)$, then $\lambda_{0}$ is an isolated eigenvalue of $A$ and $m_{a}\left(\lambda_{0}, A\right) \leq m_{a}\left(e^{\lambda_{0} t}, T(t)\right)$ where $m_{a}\left(\lambda_{0}, A\right)$ and $m_{a}\left(e^{\lambda_{0} t}, T(t)\right)$ denote, respectively, the algebraic multiplicities of $\lambda_{0}$ and $e^{\lambda_{0} t}$.

Proof of Proposition 4.1. Obviously, if $\lambda\left(t_{0}\right)=0$ for some $t_{0}>0$, then, by Lemma 4.2(1), $\lambda(t)=0$ for all $t>0$ and therefore $\sigma_{\mathrm{e}}(T(t))=\{\lambda(t)\}=\{0\}$ for all $t \in] 0, \infty\left[\right.$. This proves that $(T(t))_{t \geq 0}$ is a semigroup of Riesz type. On the other hand, for all $t \in] 0, \infty\left[, \sigma_{\mathrm{e}}(T(t))=\{0\}\right.$ implies that $T(t)$ is not invertible. Thus, by Theorem 2.1 in [13], $(T(t))_{t \geq 0}$ cannot be embedded in a $\mathrm{C}_{0}$-group and so $A$ is necessarily unbounded. By the Hille-Yosida theorem (cf. [3]), the domain of $A, D(A)$, is dense in $X$ and $\varrho(A) \neq 0$.

Let $\mu \in \varrho(A)$. Then the range of $(\mu-A)^{-1}$ is the domain of $A$. So $(\mu-A)^{-1}$ is closed and has dense range. If we suppose that its range is closed, we should have $D(A)=X$, and therefore $A$ would be bounded. Consequently, $0 \in \sigma_{\mathrm{e}}\left((\mu-A)^{-1}\right)$. To prove that $(\mu-A)^{-1}$ is a Riesz operator, it remains to show that all other elements of its spectrum are isolated eigenvalues with finite algebraic multiplicity. Note that every $0 \neq \mu \in \sigma(T(t))$ is an isolated eigenvalue of finite algebraic multiplicity. So, if $\mu^{\prime} \in \sigma(A)$ and $e^{t \mu^{\prime}}$ is an eigenvalue of $T(t)$ with finite algebraic multiplicity, then, by Lemma 4.3, $\mu^{\prime}$ is an eigenvalue of $A$ with finite algebraic multiplicity. Now let $\mu^{\prime \prime} \neq 0$ be any element of $\sigma\left((\mu-A)^{-1}\right)$. Then $\mu-1 / \mu^{\prime \prime} \in \sigma(A)$ and is an isolated eigenvalue by the preceding considerations. Hence, $\mu^{\prime \prime}$ is an eigenvalue of $(\mu-A)^{-1}$ of finite algebraic multiplicity so $(\mu-A)^{-1}$ is a Riesz operator. Now the resolvent identity, the fact that $(\lambda-A)^{-1}$ and $(\nu-A)^{-1}$ commute for all $\lambda$ and $\nu$ in $\varrho(A)$, and Theorem 3.1 of [22] show that the resolvent of $A$ is of Riesz type. 
Assume now that there exists $t>0$ such that $\lambda(t) \neq 0$. Applying Lemma 4.2(2) we conclude that $\lambda(t) \neq 0$ for all $t>0$. By Lemma 4.2 and Remark 4.2, the function $t \mapsto \lambda(t)$ is continuous. This proves that $(T(t))_{t \geq 0}$ satisfies the assumption $(\mathcal{A} 1)$ in [15] for $n=1$ and $\lambda(\cdot)=\varphi(\cdot)$. Moreover, since $D(\lambda)$ (the domain of $\lambda(\cdot)$ ) contains $] 0, \infty[$, applying Lemmas 2.4 and 2.6 of [15] we infer that $(T(t))_{t \geq 0}$ can be embedded in a strongly continuous group on $X$ and $A \in \mathcal{L}(X)$.

4.2. Hereditarily indecomposable Banach spaces. Recall that an operator in $\mathcal{L}(X)$ is said to be strictly singular if none of its restrictions to a closed infinite-dimensional subspace is an isomorphism onto its range. For the properties and the spectral theory of strictly singular operators we refer to [1] or [6]. Note however that a strictly singular operator satisfies the RieszSchauder theory of compact operators and, if $X$ is an infinite-dimensional Banach space, the class of strictly singular operators on $X, \mathcal{S}(X)$, is an ideal, in general, larger than that of compact operators.

A Banach space is said to be decomposable if it is the topological direct sum of two closed infinite-dimensional subspaces. A Banach space is said to be hereditarily indecomposable (for short an H.I. space) if it does not contain any decomposable subspace. The class of hereditarily indecomposable Banach spaces was first introduced and investigated by Gowers and Maurey in [7] (see also [4]). One of the main facts relating to this class is the following result due to Gowers and Maurey [7].

Lemma 4.4. If $X$ is a complex H.I. Banach space, then every operator in $\mathcal{L}(X)$ can be written in the form $\lambda I+S$ where $\lambda \in \mathbb{C}$ and $S \in \mathcal{S}(X)$.

The goal of this subsection is to prove the following result which gives a characterization of strongly continuous semigroups on complex H.I. Banach spaces with separable duals.

Proposition 4.2. Let $X$ be a complex H.I. Banach space and let $(T(t))_{t \geq 0}$ be a strongly continuous semigroup on $X$ with generator $A$. If $X^{\prime}$ is separable, then there are two alternatives: either $(T(t))_{t \geq 0}$ is a semigroup of strictly singular operators whose generator $A$ is unbounded with strictly singular resolvent, or else $(T(t))_{t \geq 0}$ is embeddable in a uniformly continuous group (so, its generator $A$ has the form $\beta I+S$ with $\beta \neq 0$ and $S$ strictly singular).

It is worth noticing that Proposition 4.2 shows another feature of H.I. Banach spaces with separable topological duals: the infinitesimal generator of each strongly continuous group on such spaces is necessarily bounded. Note, however, that in separable Hilbert spaces (which are not H.I. spaces), there always exist strongly continuous groups with unbounded generators. 
It suffices to apply the Stone theorem to $i A$ where $A$ is a self-adjoint operator. (Such operators exist because all separable Hilbert spaces are unitarily equivalent and therefore all classical unbounded self-adjoint operators on $L^{2}$ spaces give rise to unitarily equivalent operators on any separable Hilbert space.)

Proof of Proposition 4.2. Let $R \in \mathcal{L}(X)$ be a Riesz operator on $X$. It follows from Lemma 4.4 that $R=\beta I+S$ for some $\beta \in \mathbb{C}$ and $S \in \mathcal{S}(X)$. Then, according to [12, Theorem 3.1(i)], we have $\sigma_{\mathrm{e}}(R)=\sigma_{\mathrm{e}}(\beta I)=\{\beta\}$. But $\sigma_{\mathrm{e}}(R)=\{0\}$ (because $\operatorname{dim} X=\infty$ ), so $\beta=0$, that is, $R \in \mathcal{S}(X)$. This shows that $\mathcal{R}(X)=\mathcal{S}(X)$. Hence, to prove Proposition 4.2, it suffices to show that the hypotheses of Proposition 4.1 are satisfied. Indeed, applying Lemma 4.4 we get $T(t)=\lambda(t) I+S(t)$ where $\lambda(t) \in \mathbb{C}$ and $S(t) \in \mathcal{S}(X)$ for all $t>0$. Applying again [12, Theorem 3.1(i)] we infer that $\sigma_{\mathrm{e}}(T(t))=$ $\sigma_{\mathrm{e}}(\lambda(t) I)=\{\lambda(t)\}$. This together with the separability of $X^{\prime}$ shows that the condition $(\mathcal{A})$ holds, which completes the proof.

Note that if $X$ is a separable H.I. space, it follows from the proof above that $\mathcal{S}(X)$ and $\mathcal{R}(X)$ coincide. This fact does not require the separability of $X^{\prime}$. Now making use of Corollary 1.1 we get

Corollary 4.1. If $X$ is a separable H.I. space, then $\mathcal{S}(X)$ is a Borel subset of $\mathcal{L}_{\mathrm{S}}(X)$.

Remark 4.3. After this paper was submitted for publication, Professor G. Lancien called to our attention the paper by Räbiger and Ricker [17] which deals with strongly continuous semigroups and groups on H.I. spaces. Proposition 4.2 was established in Section 2 of that paper (see [17, Proposition 2.2 and Theorem 2.3]) without the separability hypothesis. Since our proof uses arguments from descriptive set theory, the separability hypothesis of $X$ cannot be removed (for the moment) because the space is required to be standard Borel (cf. [24, Proposition III.1.1]). On the other hand, we did not manage to drop the separability hypothesis on $X^{\prime}$ in the proof of assertion (4) of Theorem 1.1 which is used above. Nevertheless, our approach is different from that by Räbiger and Ricker, so we maintain this subsection.

4.3. Further remarks. Let $X$ be a Polish space. A subset $A$ of $X$ is called analytic if there is a Polish space $Y$ and a continuous function $f: Y \rightarrow X$ with $f(Y)=A$ (see [11]). A subset $C$ of $X$ is called coanalytic if $X \backslash C$ is analytic. The class of coanalytic subsets of $X$ is denoted by $\prod_{1}^{1}$.

Let $X$ be a Banach space and $F \in \mathcal{L}(X)$. We say that $F$ is a Fredholm perturbation if $U+F \in \Phi(X)$ whenever $U \in \Phi(X)$. The set of Fredholm perturbations is denoted by $\mathcal{F}(X)$. (Recall that $\mathcal{F}(X)$ is the greatest proper ideal of $\mathcal{L}(X)$ contained in the set of Riesz operators [1].) According to this definition one can write $F \in \mathcal{F}(X)$ if for all $U \in \mathcal{L}(X), U \in \Phi(X) \Rightarrow$ 
$U+F \in \Phi(X)$. So, if $X^{\prime}$ is separable, then $\mathcal{F}(X)$ is a coanalytic subset of $\mathcal{L}_{\mathrm{S}}(X)$. (Evidently, here we use the fact that $\Phi(X)$ is a Borel subset of $\mathcal{L}_{\mathrm{s}}(X)$ if $X^{\prime}$ is separable - cf. Corollary 1.1.) Making use of this observation, our objective here is to describe the topological complexity of some subsets of $\mathcal{L}_{\mathrm{s}}(X)$ for particular Banach spaces. Since the separability of the dual space $X^{\prime}$ is required, from the classes of spaces mentioned below, we use only Banach spaces with this property.

(1) A Banach space $X$ is said to be an $h$-space if each closed infinitedimensional subspace of $X$ contains a complemented subspace isomorphic to $X$. In [23, Theorem 6.2], Whitley proved that if $X$ is an h-space, then the ideal of strictly singular operators $\mathcal{S}(X)$ is the greatest proper ideal of $\mathcal{L}(X)$. So, as observed in [12, p. 293], we have $\mathcal{F}(X)=\mathcal{S}(X)$. This shows that $\mathcal{S}(X)$ is a coanalytic subset of $\mathcal{L}_{\mathrm{s}}(X)$ for any h-space $X$ with separable dual. This holds true, in particular, for the spaces $c, c_{0}$ and $l_{p}(1<p<\infty)$.

(2) We say that a Banach space $X$ is subprojective if given any closed infinite-dimensional subspace $M$ of $X$, there exists a closed infinite-dimensional subspace $N$ contained in $M$ and a continuous projection from $X$ onto $N$. Pfaffenberger [1, p. 100] proved that if $X$ is subprojective, then $\mathcal{F}(X)=\mathcal{S}(X)$. Accordingly, for any subprojective Banach space $X$ with separable dual, $\mathcal{S}(X)$ is a coanalytic subset of $\mathcal{L}_{\mathrm{s}}(X)$. This holds, in particular, for the spaces $c_{0}, l_{p}(1<p<\infty)$, and $L_{p}(2 \leq p<\infty)$.

(3) Let $(\Omega, \Sigma, \mu)$ be a positive measure space and let $X_{p}$ denote the space $L_{p}(\Omega, d \mu)$ with $1<p<\infty$. Since $p \in[1, \infty)$, it follows from $[16,21]$ that $\mathcal{S}\left(X_{p}\right)=\mathcal{F}\left(X_{p}\right)$. (In fact, this also holds for $p=1$ and $\infty$.) This implies that the set of strictly singular operators is a $\prod_{1}^{1}$ subset of $\mathcal{L}_{\mathrm{s}}\left(X_{p}\right)$ for $1<p<\infty$. Note that the identity $\mathcal{S}\left(X_{p}\right)=\mathcal{F}\left(X_{p}\right)$ is not specific to $L_{p}$-spaces. In fact, it is also fulfilled for $C(\Xi)$ (the Banach space of continuous scalar-valued functions on $\Xi$ with the supremum norm) provided that $\Xi$ is a compact Hausdorff space (see [16] or [21]). So, the conclusion above is also valid for $\mathcal{S}(C(\Xi))$ provided that $\Xi$ is countable.

(4) Let $X$ be a Banach space. An operator $T \in \mathcal{L}(X)$ is compact if for every bounded sequence $\left(x_{n}\right)_{n \in \mathbb{N}} \subset X$, there exists a subsequence $\left(T x_{n_{k}}\right)_{k \in \mathbb{N}}$ of $\left(T x_{n}\right)_{n \in \mathbb{N}}$ which converges in $X$. If $X$ is separable, it follows from this definition that the set of compact operators on $X, \mathcal{K}(X)$, is a $\prod_{2}^{1}$ subset of $\mathcal{L}_{\mathrm{S}}(X)$ (for the definition of $\prod_{2}^{1}$ subsets we refer to [11, p. 313]). We close this subsection by giving some examples of Banach spaces for which the ideal of compact operators belongs to the projective class $\prod_{1}^{1}$.

Recall that there are many separable Banach spaces $X$ for which $\mathcal{L}(X)$ has only one proper nonzero closed two-sided ideal. Indeed, Calkin proved that if $X$ is a separable Hilbert space, then $\mathcal{K}(X)$ is the unique proper 
nonzero closed two-sided ideal of $\mathcal{L}(X)$ (see, for example, [1, p. 81]). Gohberg, Markus and Feldman [5] established the same result for the spaces $l_{p}$, $1 \leq p<\infty$, and $c_{0}$. Accordingly, if $X$ is separable Hilbert space or one of the spaces $l_{p}, 1<p<\infty$, or $c_{0}$, then $\mathcal{K}(X)=\mathcal{F}(X)$ and therefore $\mathcal{K}(X)$ is a $\prod_{1}^{1}$ subset of $\mathcal{L}_{\mathrm{s}}(X)$.

Acknowledgments. The authors thank Professor G. Lancien who brought the reference [17] to their attention. They are also grateful to the referee for constructive remarks and questions which helped to improve the paper.

\section{References}

[1] S. R. Caradus, W. E. Pfaffenberger and B. Yood, Calkin Algebras and Algebras of Operators on Banach Spaces, Dekker, New York, 1974.

[2] J. R. Cuthbert, On semigroups such that $U(t)-I$ is compact for some $t>0$, Z. Warsch. Verw. Gebiete 18 (1971), 9-16.

[3] K. J. Engel and R. Nagel, One-Parameter Semigroups for Linear Evolution Equations, Springer, 2000.

[4] V. Ferenczi, A uniformly convex hereditarily indecomposable Banach space, Israel J. Math. 102 (1997), 199-225.

[5] I. C. Gohberg, A. Markus and I. A. Feldman, Normally solvable operators and ideals associated with them, Amer. Math. Soc. Transl. (2) 61 (1967), 63-84.

[6] S. Goldberg, Unbounded Linear Operators, McGraw-Hill, New York, 1966.

[7] W. T. Gowers and B. Maurey, The unconditional basic sequence problem, J. Amer. Math. Soc. 6 (1993), 851-874.

[8] P. R. Halmos, A Hilbert Space Problem Book, 2nd ed., Springer, New York, 1982.

[9] E. Hille and R. S. Phillips, Functional Analysis and Semigroups, Colloq. Publ. 31, Amer. Math. Soc., Providence, RI, 1957.

[10] V. I. Istrăţescu, Some remarks on a class of semigroups of operators, I, Z. Wahrsch. Verw. Gebiete 26 (1973), 241-243.

[11] A. Kechris, Classical Descriptive Set Theory, Springer, 1995.

[12] K. Latrach and A. Dehici, Fredholm, semi-Fredholm perturbations, and essential spectra, J. Math. Anal. Appl. 259 (2001), 277-301.

[13] - - - Remarks on embeddable semigroups in groups and a generalization of some Cuthbert's results, Int. J. Math. Math. Sci. 2003, no. 22, 1421-1431.

[14] K. Latrach and J. M. Paoli, Polynomially compact-like strongly continuous semigroups, Acta Appl. Math. 82 (2004), 87-99.

[15] K. Latrach, J. M. Paoli and M. A. Taoudi, A characterization of polynomially Riesz strongly continuous semigroups, submitted.

[16] V. D. Milman, Some properties of strictly singular operators, Funct. Anal. Appl. 3 (1969), 77-78.

[17] F. Räbiger and W. J. Ricker, $C_{0}$-groups and $C_{0}$-semigroups of linear operators on hereditarily indecomposable Banach spaces, Arch. Math. (Basel) 66 (1996), 60-70.

[18] J. Saint Raymond, Boréliens à coupes $K_{\sigma}$, Bull. Soc. Math. France 104 (1976), 389-406.

[19] M. Schechter, On the essential spectrum of an arbitrary operator. I, J. Math. Anal. Appl. 13 (1966), 205-215. 
[20] M. Schechter, Principles of Functional Analysis, 2nd ed., Grad. Stud. in Math. 36, Amer. Math. Soc., Providence, 2001.

[21] L. Weis, On perturbations of Fredholm operators in $L_{p}(\mu)$-spaces, Proc. Amer. Math. Soc. 67 (1977), 287-292.

[22] T. T. West, Riesz operators in Banach spaces, Proc. London Math. Soc. 16 (1966), 131-140.

[23] R. J. Whitley, Strictly singular operators and their conjugates, Trans. Amer. Math. Soc. 18 (1964), 252-261.

[24] M. Yahdi, Théorie descriptive des ensembles en géométrie des espaces de Banach, exemples, Thèse de Doctorat de Mathématiques, Université Paris 6, 1998.

Laboratoire de Mathématiques

CNRS (UMR 6620)

Université Blaise Pascal

24 avenue des Landais

63117 Aubière, France

E-mail: Khalid.Latrach@math.univ-bpclermont.fr
Département de Mathématiques Université de Corse Quartier Grossetti, BP 52 20250 Corte, France

Received April 20, 2004

Revised version April 11, 2005 